\title{
Analog Circuit Fault Diagnosis Based on Deep Learning
}

\author{
Dezan Zhao, Jun Xing \& Zhisen Wang \\ Institute of Information Science and Engineering, Dalian Polytechnic University, China
}

\begin{abstract}
Deep learning is a new field in machine learning research, whose motivation is to build neural network simulating the human brain to analyze. Stacked autoencoder, which is a style of deep learning structure, is used to solve analog circuit fault diagnosis problem. An experiment is done, whose results show that the method proposed can effectively work on analog circuit fault diagnosis using neural network model based on the deep learning theory.
\end{abstract}

KEYWORD: Deep learning; analog circuits; fault diagnosis; neural network

\section{INTRODUCTION}

Electronic circuit is the core of an electronic device, whose reliability has a direct impact on the reliability of electronic equipment. As an important part of the electronic circuit, analog circuit's fault diagnosis has an important significance. Traditional analog circuit fault diagnosis methods include element parameter identification method, fault dictionary method, approximation method, fault verification method etc. With the development of the times, the wavelet transform (Wang Shujuan et al, 2003), principal component analysis (Han Haitao et al, 2012), evolutionary algorithm (Mahdieh J et al, 2014), PSO (Sheikhan M et al, 2013), neural networks (Zhu Wenji et al, 2009) and other intelligent fault diagnosis method is applied to the analog circuits fault diagnosis.

In 2006, Hinton (Hinton $G$ E et al, 2006) proposed DBN (Deep Belief Nets) and the algorithms, which are proved efficient. The theory has made a great success in the image processing (Krizhevsky A et al, 2012), speech recognition (Shaofei Xue et al, 2014), and text recognition (Sarikaya $\mathrm{R}$ et al, 2014). This article applies the theory of deep learning to fault diagnosis, proving that the theory in fault diagnosis also has a very good performance.

\section{STACKED AUTOENCODER}

Stacked autoencoder (Yushi Chen et al, 2014) is one kind of deep neural networks which is composed of multilayer sparse encoder. Stacked autoencoder has a strong ability to express with all the advantages of deep neural networks. As used herein, the model consists of two layers sparse encoder and Softmax classified components. The specific structure is shown in Fig. 1.

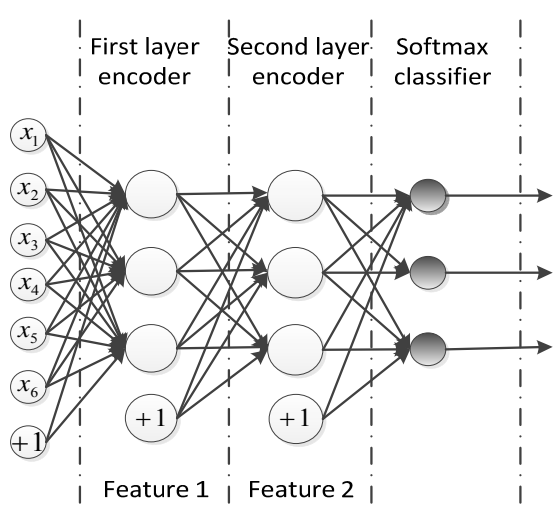

Fig.1 Stacked autoencoder

Stacked autoencoder tries to use unlabeled data to train to approaching an identity function, the input is approximately equal to the output, and training methods commonly used BP algorithm. Simple selfcoded neural network can learn to get similar lowdimensional representation of the input data just like PCA analysis. Softmax classifier, which can classify the tagged data, is extension of Logistic regression model to solve multi-classification problem.

Stack neural network adopt greedy algorithm to train one layer one time. Firstly, first layer is trained by the original input data, obtaining the weight parameters and bias parameters of first layer. Secondly, fix the parameters of the first layer and train the 
second layer using the output data of the first layer as the input data, obtaining the weight parameters and bias parameters of second layer. Finally, the softmax classifier is trained using the output data of second layer. When the train is completed, the fine tuning of entire parameters is needed using the labeled data. Fine tuning is the commonly used strategy of deep learning training, which can greatly improve the performance of stacked autoencoder. Likewise, you can use the back-propagation algorithm to fine-tune the model.

\section{SIMULATION}

The simulation experiment adopts three order bandelimination filter as the experiment subject which is shown as Fig.2.

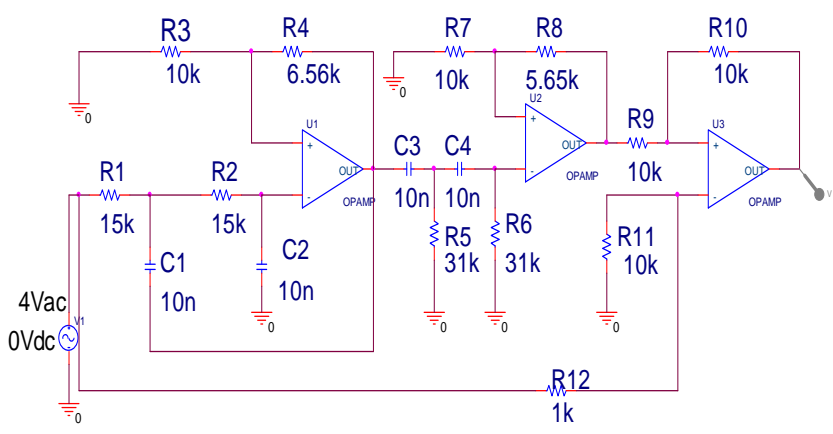

Fig.2 Three order band-elimination filter

Parameter value of each element is shown in figure 2. The normal tolerance of resistance is $5 \%$ and the normal tolerance of capacitance is $10 \%$.The resistor breaks down when the resistance value is between $5 \%$ and $50 \%$ of normal value. 3000 MonteCarlo analysis of this circuit is respectively done with the software PSPICE for five kinds of fault condition , normal status, R2 $\uparrow, \mathrm{R} 2 \downarrow, \mathrm{R} 3 \downarrow$ and R6 $\downarrow .15000$ items of data is obtained, and each item has 684 sampling point data. 10000 items is used as the training data while 5000 items is used as the test data. Some experimental data is listed in Table1.

Table 1 Some experimental data

\begin{tabular}{llllll}
\hline \multirow{2}{*}{ sample } & \multicolumn{5}{l}{ Data item } \\
\cline { 2 - 6 } & 1 & 2 & 3 & $\ldots$ & 3000 \\
\hline 1 & 1.00 & 1.00 & 1.00 & $\ldots$ & 1.00 \\
2 & 0.98 & 0.99 & 0.99 & $\ldots$ & 0.99 \\
3 & 0.97 & 0.98 & 0.99 & $\ldots$ & 0.96 \\
$\ldots$ & $\ldots$. & $\ldots$ & $\ldots$ & $\ldots$ & $\ldots$. \\
784 & 0.12 & 0.13 & 0.12 & $\ldots$ & 0.13 \\
\hline
\end{tabular}

Firstly, traditional intelligent algorithm is used for data processing. Wavelet transform is used in this article. The data firstly is processed by five layers wavelet packet decomposition, then train the BP neural network as a classifier. The accuracy rate after 20,000 iterations obtained is shown in Table 2 .

Table 2 Faulty diagnosis rate of wavelet

\begin{tabular}{|c|c|c|}
\hline code & Status classify & Accuracy rate \\
\hline $\begin{array}{llllll} & 0 & 0 & 0 & 1\end{array}$ & Normal & $32.4 \%$ \\
\hline 00010 & $\mathrm{R} 2 \uparrow$ & $64.4 \%$ \\
\hline 00100 & $\mathrm{R} 2 \downarrow$ & $85.0 \%$ \\
\hline 01000 & $\mathrm{R} 3 \downarrow$ & $71.6 \%$ \\
\hline 10000 & R6】 & $74.9 \%$ \\
\hline
\end{tabular}

Average accuracy rate of all status is $65.66 \%$ using wavelet transform as pretreatment.

Secondly, stack neural network is used to do the experiment, accuracy rate is shown in Table 3.

Table 3 Faulty diagnosis rate on different iteration numbers

\begin{tabular}{lll}
\hline \multirow{2}{*}{ Iteration } & \multicolumn{2}{l}{ Accuracy rate } \\
\cline { 2 - 3 } & No fine-tune & Have fine-tune \\
\hline 30 & $65.743 \%$ & $74.435 \%$ \\
50 & $76.660 \%$ & $83.116 \%$ \\
70 & $74.360 \%$ & $91.340 \%$ \\
90 & $78.000 \%$ & $95.040 \%$ \\
110 & $84.340 \%$ & $95.660 \%$ \\
130 & $81.980 \%$ & $96.060 \%$ \\
\hline
\end{tabular}

We can see from Table2 that accuracy of fault diagnosis is improved greatly after fine-tuning. In other words, performance of the classifier can be enhanced significantly by the strategy of fine tuning. Fault diagnosis accuracy of analog circuit based on deep learning increased significantly by comparing Table 2 and Table 3.

In fault diagnosis based on deep learning, visualization results of weight values are shown in Fig.3 and Fig.4.

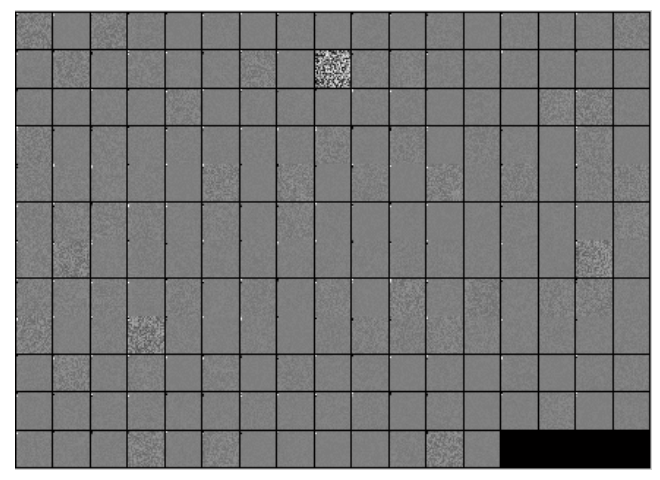

Fig.3 Visualization of first encoder 


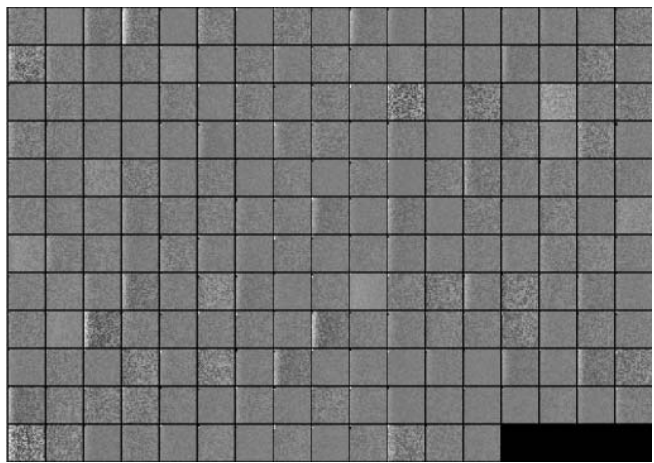

Fig.4 Visualization of second encoder

As can be seen from Fig.3 and Fig.4, the extracted feature, or representation of weight values, can be seen as the dimensionality reduction of input data.

\section{SUMMARY}

The theory of deep learning is applied to diagnose analog circuit, greatly improving the accuracy of analog circuit fault diagnosis. The more the of iterations is, the more accurate the diagnosis rate is. Also, the accuracy rate increases after fine tuning the deep neural network.

\section{REFERENCES}

Han Haitao, Ma Hongguang, Cao Jianfu. Fault Diagnosis Method of Analog Circuits Based on Characteristics of the Nonlinear Frequency Spectrum and KPCA. Transactions of China Electro technical Society [J], 2012, 27(8):248-253.

Hinton G E, Osindero S. a Fast Learning Algorithm for Deep Belief Nets [J]. Neural Computation, 2006, 18(7):15271554.

Hinton GE, Salakhutdinov RR. Reducing the Dimensionality of Data with Neural Networks [J].Science, 2006, 313(5786):504-507.

Krizhevsky A, Sutskever I, Hinton G E. Imagenet Classification with Deep Convolutional Neural Networks [J]. Advances in Neural Information Processing Systems, 2012:2012.

Mahdieh J, Farhad R. Fault Detection in Analogue Circuit Using Hybrid Evolutionary Algorithm and Neural Network [J]. Analog Integrated Circuits and Signal Processing, 2014, 80(3):551-556.

Sarikaya R, Hinton GE, Deoras. An Application of Deep Belief Networks for Natural Language Understanding. IEEE Transactions on Audio Speech and Language Processing, 2014, 22(4): 778-784.

Shaofei Xue, Abdel-Hamid. Fast Adaptation of Deep Neural Network Based on Discriminant Codes for Speech Recognition [J]. IEEE Transactions on Audio Speech and Language Processing, 2014, 22(12):1713-1725.

Sheikhan M, Shabani AA. PSO-optimized Modular Neural Network Trained by OWO-HWO Algorithm for Fault Location in Analog Circuits [J]. Neural Computing and Applications, 2013, 23(3): 519-530.

Wang Shujuan, Chen Bo. Zhao Guoliang. Analog Circuit Fault Diagnosis Based on Wavelet Packet [J]. Transactions of China Electro technical Society, 2003, 18(4): 118-122.
Yushi Chen, Zhouhan Lin, Xing Zhao. Deep Learning-Based Classification of Hyperspectral Data [J]. IEEE Journal of Selected Topics in Applied Earth Observationsand Remote Sensing. 2014, 7(6): 2107-2116.

Zhu Wenji, He Yigang. Neural Network Based Soft Fault Diagnosis of Analog Circuits With Tolerance [J]. Transactions of China Electro technical Society, 2009, 24(11):184-191. 